into two branches, was blocked separately by $3 \mathrm{~mL}$ Ropivacaine $0.5 \%$. (figure $1 \mathrm{~B}$ ) The thoracic paravertebral block at T3 with a total of $20 \mathrm{~mL}$ Ropivacaine $0.5 \%$ was performed at the sitting position by in the oblique sagittal in-plain technique from lateral to medial.

Results The patient was completely awake (Richmond agitation scale 0) during the surgery and no pain in the postoperative care unit. (NRS 0/10). The acetaminophen $1 \mathrm{~g}$ orally just once given after almost 5 hours of surgery and she was discharged home 24 hours after the surgery.

Conclusions The selective target block combinations might be considered for all structures with complex innervation, such as scapula.

\section{ULTRASOUND GUIDED ASSOCIATED TO THE FLUOROSCOPY COULD IMPROVE THE EFFICACY AND SAFETY OF THE RADIOFREQUENCY APPLICATION ON THE GANGLION IMPAR: A CASE REPORT}

${ }^{1} \mathrm{P}$ Fusco, ${ }^{2} \mathrm{~F}$ Angelucci*, ${ }^{2} \mathrm{~S}$ Tullj, ${ }^{3} \mathrm{~W}$ Ciaschi, ${ }^{2} \mathrm{~F}$ Marinangeli. ${ }^{1} 1$ Department of Anesthesia and Intensive Care, San Salvatore Academic Hospital, L'Aquila, Italy; ${ }^{2}$ University of L'Aquila, Department of Life, Health and Environmental Sciences, L'Aquila, Italy; ${ }^{3}$ Department of Anesthesia and Intensive Care, F. Spaziani Academic Hospital, Frosinone, Italy

\subsection{6/rapm-2021-ESRA.17}

Background and Aims Chronic perineal pains is a painful condition. In this study, we aimed to assess the effect the ultrasound associated to the fluoroscopy for improve the effectiveness and the safety of the radiofrequency on the ganglion impar in the patient with perianal pain ${ }^{1}$, who did not respond to standard drug therapies ${ }^{2}$

Methods The case was a 79-year-old woman with history of continuous stabbing pain in the anal region. At her first visit, the VAS was $9 / 10$.

The patient was in the prone position on the operating table.

After disinfection in the region of the sacral, a sterile echographic linear probe was placed transversely on the lower portion of the sacred until the identification of the sacral cornets.

Then, the probe is rotated in a longitudinal position until the identification of the sacrococcygea joint.Under constant ultrasound guidance and fluoroscopy, a 22 G $100 \mathrm{~mm}$ radiofrequency needle was introduced in the precoccigeo space near the ganglion impar.

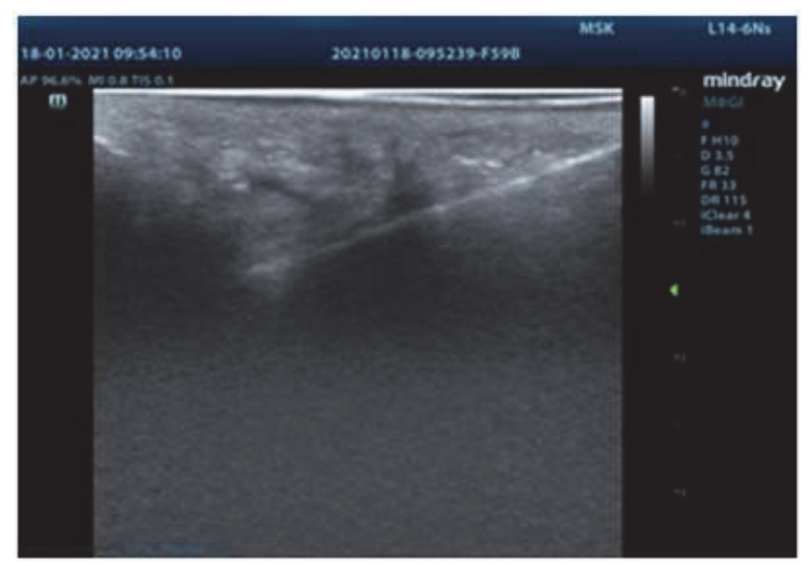

Abstract 17 Figure 1

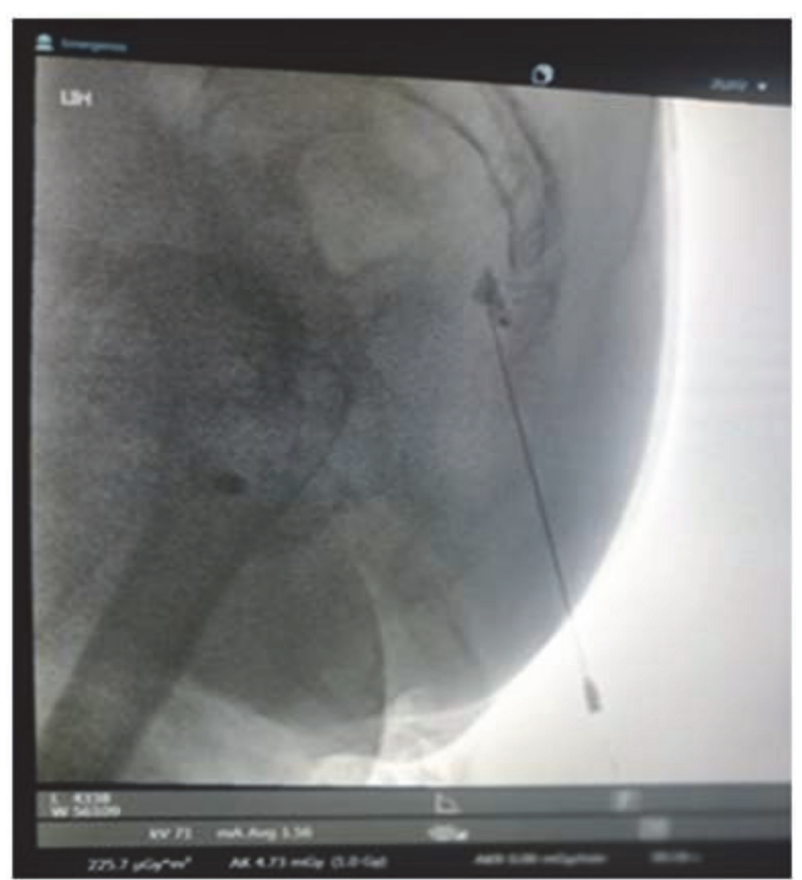

Abstract 17 Figure 2

The position of the needle tip was confirmed by injecting $1 \mathrm{~mL}$ of radiopaque dye in the retroperitoneal space.Then, a thermocouple connected to a radiofrequency generator was inserted to reproduce the patient's paresthesia via sensitive stimulation, $0.4-0.5 \mathrm{mV}$. The radiofrequency was performed at $90^{\circ} \mathrm{C}$ for 90 seconds.

Results At the end of the procedure the patient reported VAS 0 .

Conclusions The result suggests that ultrasound guided combined with fluoroscopy enhances the safety and accuracy of radiofrequency on the ganglion impar. Furthermore, it significantly reduces radiation exposure for both patients and operator.

\section{THE EFFICACY OF THE ULTRASOUND-GUIDED INTERMEDIATE CERVICAL PLEXUS BLOCK FOR CAROTID ENDARTERECTOMY}

D Mathew*, MH Wong. Khoo Teck Puat Hospital, Singapore, Singapore

10.1136/rapm-2021-ESRA.18

Background and Aims Carotid Endarterectomy (CEA) is a prophylactic surgery performed to prevent embolic stroke in patients with atheromatous disease in the carotid bifurcation. Reducing perioperative stress is critical in minimizing cardiovascular and cerebrovascular complications. Both superficial and deep cervical plexus block (CPB) have been described to provide effective analgesia and anaesthesia for head and neck surgery. Recently, an intermediate cervical plexus block has been described as a variant. We report a case that was successfully managed with ultrasound guided intermediate cervical plexus block for CEA in a high-risk patient.

Methods Patient consent was obtained for the case report. A 63-year-old male patient was scheduled for a left sided CEA with synthetic patch grafting. The patient presented with a history of right sided sensory-motor syndrome with a CT four 


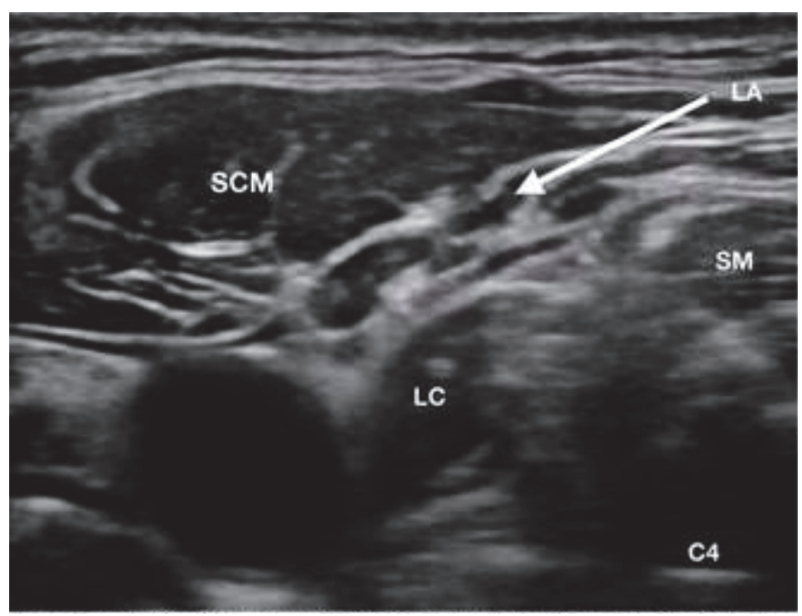

Abstract 18 Figure 1 Transverse sonogram of the lateral cervical area at the C4 level. The posterior cervical space is located between the sternocleidomastoid (SCM), longus colli (LC) and scalene (SM) muscles. Solid white arrow indicates the needle location for intermediate cervical plexus block where local anaesthetic (LA) spreads towards the carotid sheath

vessel angiogram demonstrating worsening of stenosis in the left proximal cervical segment of internal carotid artery. An ultrasound-guided Intermediate $\mathrm{CPB}$ was performed with Ropivacaine $0.4 \% 10 \mathrm{~mL}$ via a BBraun Stimuplex 22G $50 \mathrm{~mm}$ insulated needle preoperatively (figure 1). The patient opted for a general anaesthesia for the surgery. Intraoperative course was uneventful during both cross clamping of the carotid artery and internal carotid artery shunting.

Results Postoperatively, the patient was conscious, oriented, pain-free with no focal neurological deficits. The patient was monitored in surgical intensive care unit for a day and discharged to the ward.

Conclusions We demonstrated the Intermediate CPB to be feasible, effective and safe with low perioperative complications. Further studies are still required to explore this technique, including dose-finding studies to achieve optimum analgesia.

\section{ULTRASSOUND-GUIDED INFRAZYGOMATIC MAXILLARY NERVE BLOCK: AN ALTERNATIVE APPROACH TO MAXILLOFACIAL SURGERY - A CASE REPORT}

${ }^{1}$ GS Sousa* , ${ }^{2}$ A Vaz. 'Anesthesiology Resident from Centro Hospitalar Tondela-Viseu, Viseu, Portugal; ${ }^{2}$ Assistant of Anesthesiology from Centro Hospitalar Tondela-Viseu, Viseu, Portugal

\subsection{6/rapm-2021-ESRA.19}

Background and Aims Nose surgical procedures are associated with moderate postoperative pain, being the first 24 hours the most intense pain period. A lower level of postoperative pain promotes better healing, decreases anxiety and increases patient's comfort. Multimodal analgesia approach should be applied in order to provide maximum comfort while avoiding opioids adverse effects such as postoperative nausea and vomiting (PONV). PONV risk is exacerbated due to a passive blood flow that reaches the stomach during procedure. ${ }^{2}$ We report a case of an infrazygomatic maxillary nerve block (IZMNB) as part of multimodal analgesia for rhinoplasty surgery.
We intend to increase awareness to IZMNB as an effective analgesic technique for maxillofacial surgery.

Methods Case report and literature review.

Results A 28-year-old patient, ASA II, was admitted for a rhinoplasty and mentoplasty. Under general anesthesia, an ultrasound-guided IZMNB was performed bilaterally with ropivacaine $0.1 \%, 5 \mathrm{cc}$ each side, as part of a multimodal analgesic technique. Paracetamol and cetorolac were also administered. After emergence, patient referred low level of pain, needing only $2 \mathrm{mg}$ of morphine at the post-anesthetic care unit, due to chin pain (glossopharyngeal nerve area).

Conclusions IZMNB mechanism of action relies on local anesthetic dispersion within the pterygopalatine fossa. The

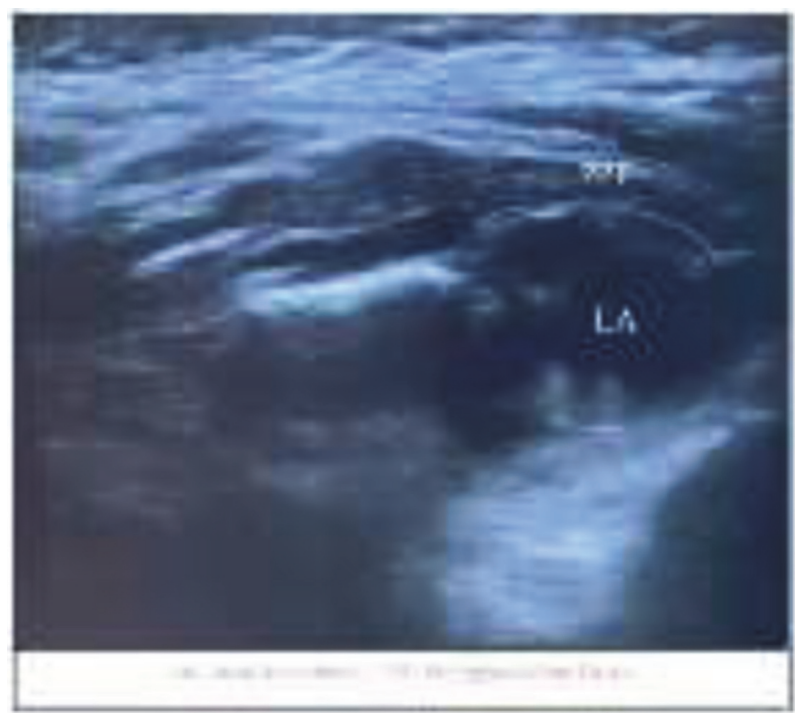

Abstract 19 Figure 1

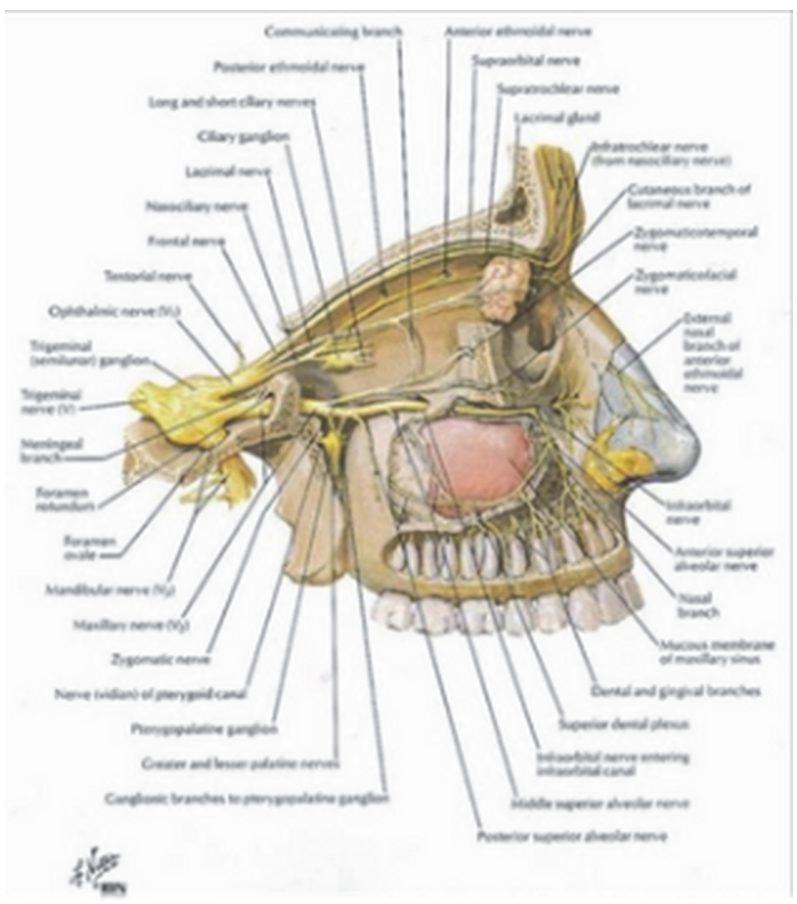

Abstract 19 Figure 2 